\title{
Clinical Trial Investigator Brochure
}

National Cancer Institute

\section{Source}

National Cancer Institute. Clinical Trial Investigator Brochure. NCI Thesaurus. Code

C115526.

Documentation that provides comprehensive data on the investigational product(s) when a study of the product(s) is using human subjects. 